\title{
A qualidade de vida de crianças durante o tratamento quimioterápico: uma revisão integrativa
}

\author{
The quality of life of children during chemotherapy treatment: an integrative review \\ La calidad de vida de los niños durante el tratamiento de quimioterapia: una revisión integradora
}

Recebido: 27/08/2021 | Revisado: 04/09/2021 | Aceito: 07/09/2021 | Publicado: 09/09/2021

\author{
Ana Carolina das Neves de Oliveira Araújo \\ ORCID: https://orcid.org/0000-0001-7969-0662 \\ Universidade Federal do Rio de Janeiro, Brasil \\ E-mail: carolnevesaraujo@gmail.com \\ Sabrina Ayd Pereira José \\ ORCID: https://orcid.org/0000-0003-1032-9259 \\ Universidade Federal do Rio de Janeiro, Brasil \\ E-mail: sabrinaayd@gmail.com \\ Thiago Privado da Silva \\ ORCID: https://orcid.org/0000-0002-7744-8319 \\ Universidade Federal do Rio de Janeiro, Brasi \\ E-mail: thiagopsilva87@gmail.com \\ Glaucia Cristina Lima da Silva \\ ORCID: https://orcid.org/0000-0001-5803-4551 \\ Universidade Federal do Rio de Janeiro, Brasil \\ E-mail: glaucia_enf@hotmail.com \\ Isis Vanessa Nazareth \\ ORCID: https://orcid.org/ 0000-0002-2504-2472 \\ Universidade Federal do Rio de Janeiro, Brasil \\ E-mail: ivnenfermagem@gmail.com
}

\begin{abstract}
Resumo
O câncer e os processos terapêuticos podem interferir, de modo negativo, na percepção da qualidade de vida relacionada a saúde (QVRS) nas crianças. Esta pesquisa tem como objetivos: analisar as produções científicas que versam sobre as intervenções utilizadas para melhorar a qualidade de vida de crianças com câncer durante o tratamento quimioterápico. $\mathrm{O}$ método do estudo utilizado foi a revisão integrativa de literatura, o qual foram realizadas seis etapas do processo de elaboração, onde as buscas foram nas bases de dados: MEDLINE, CINAHL e Scopus, utilizando os descritores qualidade de vida, câncer, crianças, quimioterapia nos idiomas português e inglês. $\mathrm{Na}$ busca foram encontrados 479 artigos, onde selecionou-se 21 publicações científicas, que estavam de acordo com a temática estabelecida. As intervenções encontradas nas produções científicas para melhorar a qualidade de vida de crianças com câncer durante o tratamento quimioterápico são: uso de medicamentos, exercício físico e yoga. Observou-se a necessidade da publicação de novos artigos, bem como de produções brasileiras atualizadas acerca do tema, visando proporcionar uma melhora na qualidade de vida das crianças em tratamento quimioterápico.
\end{abstract}

Palavras-chave: Qualidade de vida; Neoplasias; Criança; Quimioterapia.

\begin{abstract}
Cancer and therapeutic processes can negatively interfere with the perception of health-related quality of life (HRQOL) in children. This research aims to analyze scientific productions that deal with interventions used to improve the quality of life of children with cancer during chemotherapy. The study method used was the integrative literature review, which performed six stages of the elaboration process, where the searches were in the databases: MEDLINE, CINAHL and Scopus, using the descriptors quality of life, cancer, children, chemotherapy in Portuguese and English. In the search, 479 articles were found, where 21 scientific publications were selected, which were in accordance with the established theme. The interventions found in scientific productions to improve the quality of life of children with cancer during chemotherapy treatment are: use of medication, physical exercise and yoga. There was a need to publish new articles, as well as updated Brazilian productions on the subject, aiming to improve the quality of life of children undergoing chemotherapy.
\end{abstract}

Keywords: Quality of life, Neoplasms; Child; Drug therapy.

\section{Resumen}

El cáncer y los procesos terapéuticos pueden interferir negativamente con la percepción de la calidad de vida relacionada con la salud (CVRS) en los niños. Esta investigación tiene como objetivo analizar las producciones científicas que abordan las intervenciones utilizadas para mejorar la calidad de vida de los niños con cáncer durante la quimioterapia. El método de estudio utilizado fue la revisión integradora de la literatura, que realizó seis etapas del 
proceso de elaboración, donde las búsquedas fueron en las bases de datos: MEDLINE, CINAHL y Scopus, utilizando los descriptores calidad de vida, cáncer, niños, quimioterapia en portugués e inglés. En la búsqueda se encontraron 479 artículos, donde se seleccionaron 21 publicaciones científicas, que estuvieron de acuerdo con el tema establecido. Las intervenciones encontradas en producciones científicas para mejorar la calidad de vida de los niños con cáncer durante el tratamiento de quimioterapia son: uso de medicación, ejercicio físico y yoga. Era necesario publicar nuevos artículos, así como producciones brasileñas actualizadas sobre el tema, con el objetivo de mejorar la calidad de vida de los niños sometidos a quimioterapia.

Palabras clave: Calidad de vida; Neoplasias; Niño; Quimioterapia.

\section{Introdução}

O câncer é uma doença que até os dias atuais gera grande impacto à vida do indivíduo, remetendo à dor e sofrimento. Mesmo com todos os avanços científicos na área da saúde, a doença é temida e associada à morte. Apesar de estar diante de inovações envolvendo a tecnologia dura e leve, esta doença causa impactos físicos muito agressivos que, consequentemente, causam grandes dores emocionais ao paciente, bem como ao seu corpo familiar (Elias, Moreira \& Parra, 2017).

O Instituto Nacional do Câncer (Brasil, 2016) estimou que em 2019, ocorria no Brasil 420 mil novos casos de câncer, sendo as neoplasias pediátricas responsáveis por um número de 3\% a 10\% desse total. Nos países em desenvolvimento, o câncer infantil representa a segunda maior causa de morte após o primeiro ano de vida. Independente da faixa etária, o diagnóstico da doença sempre é um momento difícil. A descoberta do câncer infantil repercute nocivamente na vida dos pais, familiares e da própria criança, impactando-os com sentimento de pesar, angústia, medo da morte, depressão, desespero e estigmas (Castro, 2010).

A doença oncológica percorre uma longa jornada até que seu diagnóstico seja definido, os sinais e sintomas não são necessariamente específicos, o que pode causar um diagnóstico equivocado devido ao fato de ser confundido com outras doenças da infância. Por isso, muitas crianças são encaminhadas ao centro de tratamento com a doença em estágio avançado. O medo do diagnóstico, a desinformação dos pais e a dificuldade com o acesso ao serviço de saúde podem contribuir para o diagnóstico tardio do câncer, muitas vezes, comprometendo a qualidade de vida (QV) das crianças (Fermo et al., 2014).

Corroborando com os aspectos relacionados ao enfrentamento da doença, o tratamento do câncer infantil, geralmente, requer longos períodos de internação, além de várias intervenções terapêuticas, o que torna necessária e de fundamental importância uma atenção especial para as questões sociais e psicológicas das crianças. O direito de brincar e a evolução física, afetiva, social e pessoal devem ser preservados, para que o atendimento da equipe seja cada vez melhor, visando a promoção de uma QV satisfatória (Barros et al.,2017).

A Organização Mundial da Saúde (OMS, 1995), define qualidade de vida como uma percepção que o próprio indivíduo tem sobre sua posição na vida, num contexto cultural, em um sistema de valores em que está enquadrado, de acordo com seus padrões, objetivos, expectativas e preocupações. A QV da criança pode sofrer algumas alterações devido ao surgimento do câncer, pois implica em modificações da rotina diária e até o afastamento do convívio escolar, familiar e social para a realização do tratamento, que por vezes pode ser doloroso e alterar sua aparência física (Vieira et al., 2009).

Existem modalidades de tratamento para controle e/ou combate do câncer infantil, que são: quimioterapia, radioterapia, transplante de medula óssea ou transplante de células hematopoiética, essas formas de tratamento são aplicadas dependendo do tipo de tumor e de acordo com a extensão da doença. No entanto, a quimioterapia é a modalidade mais utilizada, podendo ser associada ou não às outras modalidades de tratamento (Souza et al., 2017).

A quimioterapia é uma fase crucial para o tratamento do câncer, sabe-se que ela tem aumentado a sobrevida dos pacientes, no entanto, atinge, indiscriminadamente, todas as células dos organismos e, devido à sua toxicidade, acaba causando alguns efeitos adversos que podem influenciar na QV da criança (Almico \& Faro 2014). Náuseas, vômitos, diarreia, alopécia, mucosite, dor e fadiga são alguns dos efeitos colaterais mais angustiantes que surgem durante e após o tratamento 
quimioterápico, o que afeta de forma negativa a QV, pois podem impedir a realização das atividades infantis diárias (Giagnuolo et al., 2019).

O câncer e os processos terapêuticos podem interferir, de modo negativo, na percepção da qualidade de vida relacionada à saúde (QVRS), por isso, a sua avaliação é considerada um parâmetro importante na área da oncologia (Matos et al., 2017). Portanto, a QVRS é um resultado importante para avaliar o impacto do câncer na criança (Richter et al., 2018). O câncer tem efeitos adversos na QV da criança, dessa forma, medir a QV tornou-se parte integrante dos cuidados de saúde (Santos et.al., 2016). Diante do pressuposto, o objetivo do estudo foi analisar as produções científicas que versam sobre as intervenções utilizadas para melhorar a qualidade de vida de crianças com câncer durante o tratamento quimioterápico.

\section{Metodologia}

O método do estudo utilizado foi a revisão integrativa de literatura, na qual foram realizadas seis etapas do processo de elaboração, a saber: identificação do tema e seleção da hipótese ou questão de pesquisa para elaboração da revisão integrativa; estabelecimento de critérios para inclusão e exclusão de estudos/amostragem ou busca na literatura; definição das informações a serem extraídas dos estudos selecionados/categorização dos estudos; avaliação dos estudos incluídos na revisão integrativa; interpretação dos resultados ; e apresentação da revisão/síntese do conhecimento (Mendes, Silveira \& Galvão, 2008).

A questão que norteou a pesquisa foi: quais as intervenções utilizadas para melhorar a qualidade de vida de crianças com câncer durante o tratamento quimioterápico? A busca dos estudos foi realizada nos meses de agosto a novembro de 2019. Foram utilizadas as bases de dados eletrônicas Medical Literatureend Retrieval System online (MEDLINE), Cumulative Index to Nursing and Allied Health Literature (CINAHL), e Scopus. Para a busca nas bases de dados foram aplicados os Descritores em Ciências da Saúde (DeCS): qualidade de vida, câncer, crianças, quimioterapia, em português; e em inglês, qualityoflife, neoplasms, childrens, drugtherapy. No pareamento dos descritores, foram aplicados os operadores booleanos AND e OR. A busca foi realizada pelo acesso online nas bases de dados selecionadas, de forma independente, por dois revisores.

Na seleção dos artigos, adotaram-se os seguintes critérios de inclusão: artigos que apresentassem a temática da qualidade de vida em crianças durante o tratamento quimioterápico e que contemplassem os objetivos do estudo, com disponibilidade de texto on-line na íntegra. Os critérios de exclusão utilizados foram: artigos que não se encaixavam na referida temática, bem como editoriais, resumos de anais, relatórios de gestão e boletins epidemiológicos. Nessa pesquisa não foi delimitado o recorte temporal, com intuito de apurar, nas bases de dados, o início da produção científica e difusão da temática em questão.

A busca inicial foi realizada a partir da utilização dos descritores em Ciências da Saúde (DeCS), onde, em seguida, aplicaram-se filtros para o refinamento da pesquisa em cada uma das bases de dados com a utilização das ferramentas eletrônicas disponíveis em portais eletrônicos, onde se considerou acesso gratuito e texto completo. Na sequência foi realizada a leitura dos títulos e resumos. O processo de busca e seleção dos estudos seguiram as recomendações do Preferred Reporting Items for Systematic Reviewsand Meta-Analyses (PRISMA)(Galvão, Pansani\&Harrad, 2015), que consiste em um checklist que contém um fluxograma de quatro etapas (identificação, seleção, elegibilidade e inclusão), podendo ser usado como uma base para relatos de revisões sistemáticas de outros tipos de pesquisa, particularmente avaliações de intervenções.

$\mathrm{Na}$ busca foram encontrados 479 artigos, 10 deles estavam duplicados e foram excluídos manualmente após a identificação dos mesmos, restando 469 artigos que posteriormente foram lidos os títulos e resumos, onde foram préselecionados 139 para leitura na integra. Dos 139 artigos selecionados, 118 foram excluídos por não responderem à questão do estudo. Após leitura dos artigos foram selecionadas 21 publicações científicas, que estavam de acordo com a temática estabelecida, demonstradas na Figura 1. 
Figura 1: Fluxo do processo de seleção dos estudos para a revisão integrativa de literatura, elaborado com base nas recomendações PRISMA.

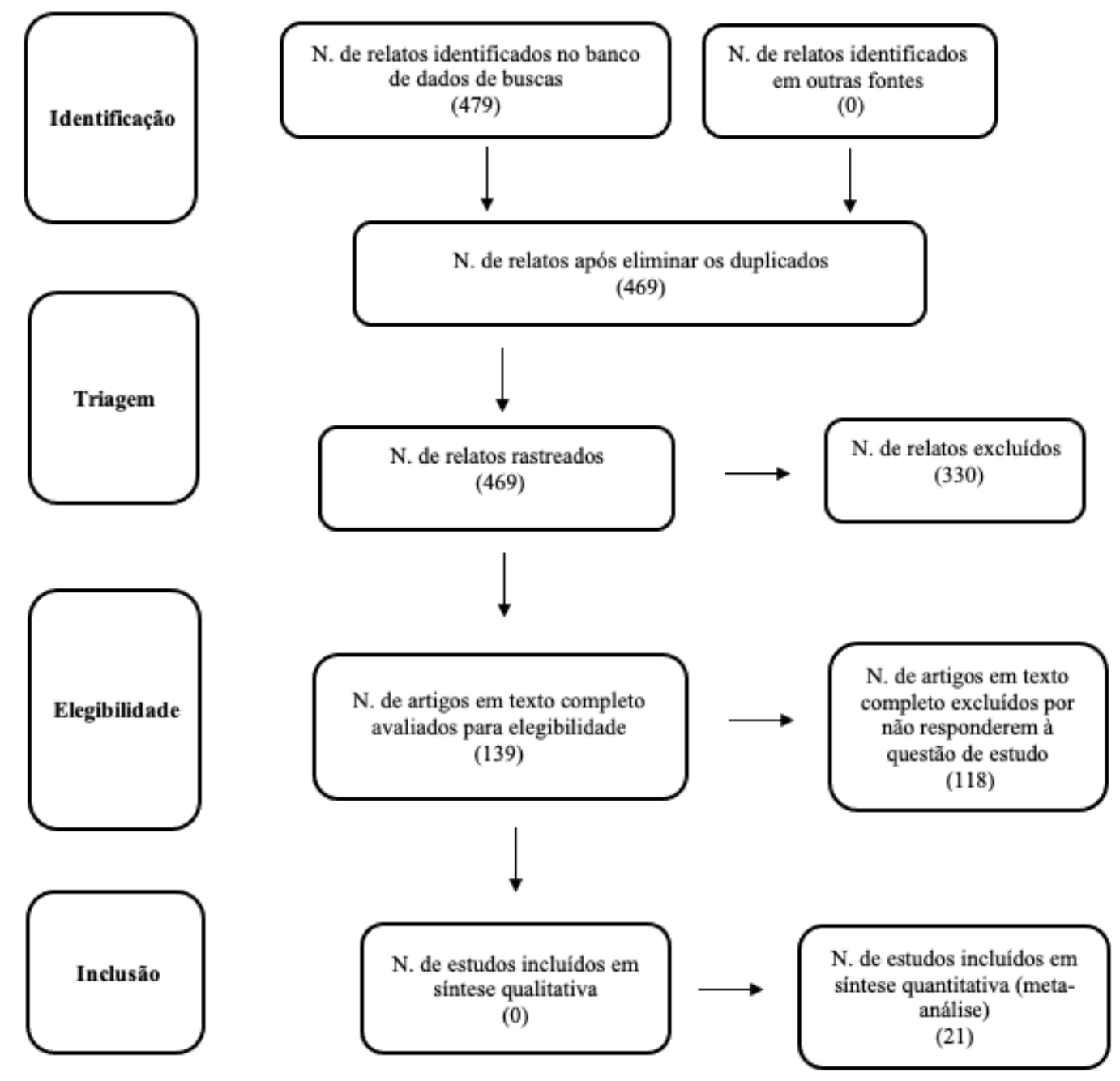

Fonte: Autores (2020).

$\mathrm{Na}$ análise do estudo, elaborou-se um instrumento no Software Microsoft Excel, de maneira a organizar um banco de dados sobre os artigos encontrados. Este instrumento contém os seguintes itens: código de identificação, base de dados que o artigo está indexado, ano da publicação, país, título do artigo, autores, e por último, o nível de evidência. Os dados obtidos foram tratados estatisticamente através da distribuição de frequências, utilizando a planilha eletrônica onde foi criado o instrumento. Para classificação do nível de evidência (NE), foram considerados sete níveis, segundo a classificação de Melnyk (Melnyk\&Fineout-Overholt, 2011), apresentada no Quadro 1. 
Quadro 1: Níveis de Evidência segundo Classificação de Melnyk. / NE* - Nível de Evidência.

\begin{tabular}{|c|l|}
\hline NE & TIPOS DE ESTUDO \\
\hline I & $\begin{array}{l}\text { Evidência proveniente de revisões sistemáticas ou metanálises de todos os ensaios clínicos randomizados controlados (ECRC) } \\
\text { relevantes ou oriundas de diretrizes clínicas baseadas em revisões sistemáticas de ECRC }\end{array}$ \\
\hline II & Evidência derivada de pelo menos um ECRC bem delineado; \\
\hline III & Evidência obtida de ensaios clínicos bem delineados sem randomização; \\
\hline IV & Evidência proveniente de estudo de caso controle ou estudo de coorte bem delineado; \\
\hline V & Evidência proveniente de revisões sistemáticas de estudos qualitativos e descritivos; \\
\hline VI & Evidência derivada de um estudo descritivo ou qualitativo; \\
\hline VII & Evidência oriunda da opinião de autoridades e/ou relatórios de comitês de especialistas. \\
\hline
\end{tabular}

NE* - Nível de Evidência. Fonte: Melnyk e Fineout-Overholt (2011).

Portanto, é importante salientar que, com base no tipo de delineamento de estudo, e na sua capacidade de afirmar causa e efeito, são consideradas evidências fortes, os níveis I e II, evidências moderadas III a IV, e os níveis V a VII são evidências fracas (Melnyk\&Fineout-Overholt, 2011). Em relação aos aspectos éticos e legais, por se tratar de um estudo com metodologia baseada em uma pesquisa bibliográfica, somado ao fato que não houve conflito de interesses, não houve necessidade da submissão desta revisão integrativa ao Comitê de Ética em Pesquisa.

\section{Resultados}

Dos 21 artigos selecionados, estão indexados, em sua maioria, na base de dados MEDLINE com 09 artigos (42,85\%) ao total. Seguido de 08 artigos $(38,1 \%)$ na CINAHL e 04 artigos $(19,05 \%)$ na Scopus. O quadro 02 expõe os resultados encontrados após a pesquisa.

Quadro 2: Resultados selecionados após a pesquisa.

\begin{tabular}{|c|c|c|c|c|}
\hline CÓDIGO & BASE & ANO & PAÍS & TÍTULO \\
\hline P1 & CINAHL & 2006 & EUA & $\begin{array}{l}\text { Double-blind, placebo-controlled study of quality of life, hematologic end } \\
\text { points, and safety of weekly epoetin alfa in children with cancer receiving } \\
\text { myelosuppressive chemotherapy. }\end{array}$ \\
\hline P2 & CINAHL & 2009 & EUA & $\begin{array}{l}\text { Aggressive Treatment of Nonmetastatic Osteosarcoma Improves Health- } \\
\text { Related Quality of Life in Children and Adolescents. }\end{array}$ \\
\hline P3 & CINAHL & 2017 & EUA & $\begin{array}{l}\text { Glutamine for the treatment of vincristine-induced neuropathy in children } \\
\text { and adolescents with cancer. }\end{array}$ \\
\hline P4 & CINAHL & 2012 & EUA & $\begin{array}{l}\text { Filgrastim vs pegfilgrastim: uma questão de qualidade de vida para } \\
\text { crianças. }\end{array}$ \\
\hline P5 & CINAHL & 2014 & EUA & $\begin{array}{l}\text { Outpatient versus inpatient IV antibiotic management for pediatric } \\
\text { oncology patients with low risk febrile neutropenia: A randomised trial. }\end{array}$ \\
\hline P6 & CINAHL & 2011 & CHINA & $\begin{array}{l}\text { Effect of Chinese drugs combining with chemotherapy on quality of life in } \\
146 \text { children with solid tumor. }\end{array}$ \\
\hline P7 & CINAHL & 2017 & EUA & $\begin{array}{l}\text { Evaluation of aprepitant for acute chemotherapy-induced } \\
\text { nausea and vomiting in children and adolescents with acute lymphoblastic } \\
\text { leukemia receiving high-dose methotrexate. }\end{array}$ \\
\hline P8 & CINAHL & 2012 & EUA & $\begin{array}{l}\text { Pilot Study of the Effect of Romiplostim on Child Health-Related Quality } \\
\text { of Life (HRQoL) and Parental Burden in Immune Thrombocytopenia } \\
\text { (ITP). }\end{array}$ \\
\hline
\end{tabular}


Research, Society and Development, v. 10, n. 11, e547101119946, 2021

(CC BY 4.0) | ISSN 2525-3409 | DOI: http://dx.doi.org/10.33448/rsd-v10i11.19946

\begin{tabular}{|c|c|c|c|c|}
\hline P9 & SCOPUS & 2015 & EUA & $\begin{array}{l}\text { Patient-Reported Outcome Coordinator Did Not Improve Quality of Life } \\
\text { Assessment Response Rates: A Report from the Children's Oncology } \\
\text { Group. }\end{array}$ \\
\hline P10 & SCOPUS & 2019 & EUA & $\begin{array}{l}\text { Single center experience on efficacy and safety of Aprepitant for } \\
\text { preventing chemotherapy-induced nausea and vomiting (CINV) in } \\
\text { pediatric Hodgkin Lymphoma. }\end{array}$ \\
\hline P11 & SCOPUS & 2017 & $\begin{array}{l}\text { REINO } \\
\text { UNIDO }\end{array}$ & $\begin{array}{l}\text { Prospective evaluation of quality of life in children treated in UKALL } 2003 \\
\text { for acute lymphoblastic leukaemia: A cohort study. }\end{array}$ \\
\hline P12 & SCOPUS & 2011 & EUA & $\begin{array}{l}\text { Health-related quality of life anticipated with different management } \\
\text { strategies for paediatric febrile neutropaenia. }\end{array}$ \\
\hline $\mathrm{P} 13$ & MEDLINE & 2016 & EUA & $\begin{array}{l}\text { Physical exercise training interventions for children and young adults } \\
\text { during and after treatment for childhood câncer. }\end{array}$ \\
\hline $\mathrm{P} 14$ & MEDLINE & 2015 & EUA & $\begin{array}{l}\text { Prospective, Longitudinal Assessment of Quality of Life in Children from } \\
\text { Diagnosis to Three Months off Treatment for Standard Risk (SR) Acute } \\
\text { Lymphoblastic Leukemia (ALL): Results of Children's Oncology Group } \\
\text { Study AALL0331. }\end{array}$ \\
\hline $\mathrm{P} 15$ & MEDLINE & 2015 & EUA & $\begin{array}{l}\text { Children's Perspective on Health-Related Quality of Life during Active } \\
\text { Treatment for Acute Lymphoblastic Leukemia: An Advanced Content } \\
\text { Analysis Approach. }\end{array}$ \\
\hline P16 & MEDLINE & 2011 & HOLANDA & $\begin{array}{l}\text { Impaired sleep affects quality of life in children during maintenance } \\
\text { treatment for acute lymphoblastic leukemia: an exploratory study. }\end{array}$ \\
\hline $\mathrm{P} 17$ & MEDLINE & 2008 & HOLANDA & $\begin{array}{l}\text { Effect of dexamethasone on quality of life in children with acute } \\
\text { lymphoblastic leukaemia: a prospective observational study. }\end{array}$ \\
\hline $\mathrm{P} 18$ & MEDLINE & 2017 & EUA & $\begin{array}{l}\text { Voices of children and adolescents on phase } 1 \text { or phase } 2 \text { cancer trials: A } \\
\text { new trial endpoint? }\end{array}$ \\
\hline P19 & MEDLINE & 2015 & EUA & $\begin{array}{l}\text { A pilot study to evaluate the feasibility of individualized yoga for inpatient } \\
\text { children receiving intensive chemotherapy. }\end{array}$ \\
\hline $\mathrm{P} 20$ & MEDLINE & 2016 & EUA & $\begin{array}{l}\text { Pediatric quality of life in long-term survivors of childhood cancer treated } \\
\text { with anthracyclines. }\end{array}$ \\
\hline $\mathrm{P} 21$ & MEDLINE & 2014 & BRASIL & $\begin{array}{l}\text { Supplementation with Selenium Can Influence Nausea, Fatigue, Physical, } \\
\text { Renal, and Liver Function of Children and Adolescents with Cancer. }\end{array}$ \\
\hline
\end{tabular}

Fonte: Autores (2020).

Quanto ao ano de publicação, em ordem de maior para a menor frequência, 04 artigos (19,05\%) em 2015, 04 $(19,05 \%)$ em 2017, 03 (14,3\%) em 2011, 02 (9,52\%) em 2014, 02 (9,52\%) em 2012, 02 (9,52\%) em 2016, 01 (4,76\%) em 2006, 01 (4,76\%) em 2008, 01 (4,76\%) em 2009 e 01 (4,76\%) em 2019. Quanto ao idioma em que os artigos foram publicados, todos os 21 (100\%) estudos selecionados foram em inglês. Com relação ao País de publicação, temos os Estado Unidos da América com 16 (76,2\%) publicações, seguido de Holanda com 02 (9,52\%), Reino Unido com 01 (4,76\%), China com 01 $(4,76 \%)$ e Brasil com 01 (4,76\%) artigo.

Entre os artigos selecionados, as 21 produções científicas são de metodologia quantitativa (100\%). Dentre os tipos de estudos quantitativos, apresentam-se: 08 estudos clínicos controlados randomizados (38,1\%), 01 estudo longitudinal descritivo (4,76\%), 01 estudo de coorte retrospectivo (4,76\%), 01 estudo prospectivo longitudinal (4,76\%), 01 estudo de coorte prospectivo (4,76\%), 01 estudo longitudinal (4,76\%), 01 estudo retrospectivo não randomizado (4,76\%), 01 estudo prospectivo $(4,76 \%), 01$ estudo transversal (4,76\%), 01 estudo cruzado randomizado (4,76\%), 04 estudos quantitativos exploratórios 
$(19,06 \%)$. No que se refere à força de evidência dos artigos, estes foram classificados com maior frequência em evidência fraca, com 11 publicações (52,38\%), evidência forte, com 08 estudos $(38,1 \%)$ e evidência moderada com 02 artigos $(9,52 \%)$, e estão representados na Tabela 1.

Tabela 1. Classificação dos artigos selecionados nesta revisão, de acordo com o nível de evidência

$\begin{array}{ccc}\text { NIVEL DE } & \mathbf{n} & \boldsymbol{\%} \\ \text { EVIDÊNCIA } & & 0 \\ \text { I } & 0 & 38,1 \% \\ \text { II } & 8 & 0 \\ \text { III } & 0 & 9,52 \% \\ \text { IV } & 2 & 0 \\ \text { V } & 0 & 52,38 \% \\ \text { VI } & 11 & 0 \\ \text { VII } & 0 & \mathbf{1 0 0 \%} \\ \text { TOTAL } & \mathbf{2 1} & \end{array}$

Fonte: Autores (2020).

Dos 21 artigos analisados, 10 falam sobre os efeitos causados pela quimioterapia, como; náusea, vômito, fadiga e dor, além da neutropenia febril, que podem contribuir de forma negativa para a QV. Outros 02 estudos mostram que efeitos neurológicos, como ansiedade e depressão, influenciam na QV; 01 estudo relata sobre a distúrbio do sono durante o tratamento do câncer; 06 artigos apontam sobre o uso de medicamentos que podem ajudar na QV de crianças que são submetidas à quimioterapia; 01 estudo discorre sobre a importância da realização da atividade física; e 01 estudo fala sobre a realização da yoga durante o tratamento quimioterápico. Esses dois últimos estudos são formas não farmacológicas que podem contribuir de forma positiva na melhora da QV das crianças que são tratadas com quimioterapia.

Para uma melhor visualização e interpretação da qualidade de vida durante o tratamento quimioterápico apresentados nos artigos, optou-se por categorizar as estratégias e influências encontradas em: influência dos efeitos adversos da quimioterapia na QV e ações interventivas na QV, conforme apontados respectivamente na Tabela 2 e na Tabela 3.

Tabela 2: Ações interventivas utilizadas para melhora da QV.

\begin{tabular}{cc|} 
Ações interventivas na QV & $\mathbf{n}^{*}$ \\
\hline Medicamentos & 6 \\
\hline Exercício Físico & 1 \\
Yoga & 1 \\
\hline
\end{tabular}

*n: número de artigos que citaram. Fonte: Autores (2020). 
Tabela 3: Categorias das influências dos efeitos adversos da quimioterapia que influenciam na QV.

\section{Influência dos efeitos adversos da n* \\ quimioterapia na QV}

\begin{tabular}{cc} 
Efeitos neurológicos & 2 \\
\hline Distúrbio de Sono & 1 \\
\hline Efeitos sistêmicos & 10
\end{tabular}

*n: número de artigos que citaram. Fonte: autores (2020).

\section{Discussão}

Os dados apontam que as discussões acerca da temática da QV durante o tratamento quimioterápico são de baixa evidência científica, contribuindo para uma má tomada de decisões e intervenções da prática clínica, afetando diretamente o cuidado e influenciando negativamente na QV das crianças submetidas à quimioterapia. Sabe-se que na modalidade de tratamento para o câncer infantil, a quimioterapia é o principal recurso contra a doença. Os autores são unânimes ao descreverem os principais efeitos colaterais da quimioterapia. Estudos mostram que as reações adversas mais presentes descritas são náuseas, vômitos, fadiga, dor e alopecia (Ribeiro, Fonseca, Almeida, Rocha \& Oliveira, 2015).

Devido à particularidade que distingue cada indivíduo em tratamento quimioterápico, os artigos pontuam que os efeitos adversos da quimioterapia influenciam de forma negativa na qualidade de vida das crianças (Braam, et.al, 2016; Eiser, et.al, 2017; Orme et al., 2014; Shi, Tian, Zhu, Wang \&Qin, 2011; Klaassen, et.al, 2012). Evidências científicas apontam que náuseas, diarreia e vômitos foram, respectivamente, as principais reações decorrentes da quimioterapia em relação à toxicidade gastrintestinal apresentada pelos pacientes, podendo afetar a condição nutricional, o equilíbrio hidroeletrolítico e a qualidade de vida (National Comprehensive Cancer Network, 2015).

Acredita-se que o desconforto proporcionado ao paciente pela náusea e pelo vômito contribui para a diminuição da qualidade de vida, visto que os pacientes se tornam debilitados e sem disposição para conduzirem adequadamente os seus afazeres diários (Orme et al., 2014; Eiser, et.al., 2017). A fadiga e a dor foram efeitos adversos apontados nas produções científicas que influenciam de forma negativa na QV (Mitchell, et.al, 2016; Ryerson, et.al, 2016). A fadiga, relacionada ao câncer, tem sido descrita como o sintoma prevalente em pacientes oncológicos pediátricos, ocorrendo entre $36 \%$ a $93 \%$ dos casos, com maior nível de fadiga experimentado por aqueles submetidos ao tratamento quimioterápico. De acordo com um estudo, a utilização do Selenium pode ajudar na melhora da fadiga, consequentemente, melhorando a QV desse paciente (Moraiset.al., 2018; Rodrigues, Martin \& Moraes, 2016).

Estudos mostram que a dor relacionada ao câncer pode ocorrer devido à invasão tumoral nos ossos, vísceras e sistema nervoso; por espasmos musculares, linfedema, úlceras de decúbito; ou pelo próprio tratamento e complicações (Monteiro, et al., 2014). O manejo da dor é considerado um princípio básico para a qualidade de vida, mas em crianças, seu tratamento é bastante crítico e sensível, em virtude da dificuldade de mensurar a grandeza da dor nessa faixa etária (Andersen et al., 2014).

Os dados mostram que a neutropenia febril (NF) é um dos efeitos que acometem frequentemente algumas crianças submetidas à quimioterapia. A NF pode ser também uma complicação da própria doença, como nos casos das neoplasias hematológicas. Além disso, afeta negativamente a qualidade de vida, predispondo os pacientes a internações e isolamento hospitalar, pois é necessária a hospitalização para receber antibióticos intravenosos, o que acaba gerando um impacto emocional e financeiro nas famílias, devido à perda de trabalho e à interrupção da vida familiar (Braam et al., 2016; Orme et al., 2014; Shi et al., 2011; Klaassen, et.al, 2012).

Distúrbios do sono também podem afetar a qualidade de vida de crianças em tratamento quimioterápico. Um estudo relata que na maioria das vezes o distúrbio está relacionado à ansiedade e ao estresse do tratamento, que atraso no início do 
sono, sonolência diurna e despertar noturno contribuem para o comprometimento do mesmo, assim afetando diretamente a QV (Vries, et.al., 2008). De acordo com os estudos, o tratamento quimioterápico se junta a diversos sentimentos que fazem parte do cotidiano da criança sob tratamento. Como, por exemplo, o medo, a tristeza, o desânimo, a desesperança, o pesar, a aflição, a angústia, a inquietação e a impaciência. Estes sentimentos surgem em reação ao impacto do diagnóstico, à incerteza quanto ao prognóstico e à complexidade que envolve o tratamento quimioterápico (Almico \& Faro, 2014).

Os estudos mostram que a ansiedade e depressão estão presentes no dia a dia da criança sob tratamento quimioterápico (Johnston, et.al., 2015; Mitchell, et.al., 2016). Tendo em vista esses resultados, destaca-se a importância da identificação e da abordagem multiprofissional da ansiedade e da depressão nos serviços de oncologia, enfocando não apenas os sofrimentos ocasionados por essas condições psiquiátricas, mas também sobre os seus efeitos nos diferentes domínios da qualidade de vida da pessoa com câncer. A detecção precoce e a intervenção eficaz junto às pessoas com sintomas de ansiedade e depressão podem reduzir o custo humano do câncer, não só para os pacientes, mas também para os cuidadores e/ou familiares (Agarwal \&Maroko-Afek, 2018).

Em relação à terapia medicamentosa, no estudo, foi possível observar que o uso de determinados medicamentos pode vir a contribuir para uma melhora da QV das crianças em tratamento quimioterápico (Felix- Ukwu, Reichert, Bernhardt, Schafer \& Berger, 2017). De acordo com os estudos, alguns fármacos utilizados em casos de crianças acometidas pela NF, como Cefepima, peffilgrastim e filgrastim contribuíram para uma melhora significativa da QV desses pacientes, o que leva a menos hospitalizações e menos interrupções para a criança e família (Orme et al.,2014; Sands, et.al., 2017).

As evidências científicas mostram que o uso do aprepitant e selenium contribui para uma melhora da QV dos pacientes pediátricos, pois este medicamento colabora na redução de náuseas e vômitos induzidos pela quimioterapia (Morais et al., 2018; Orme et al., 2014). No universo dos estudos, uma produção científica mostrou que os glicocorticóides podem ajudar de forma positiva na QV, reduzindo os efeitos adversos da quimioterapia (Hinds, et.al., 2017).

As intervenções farmacológicas usadas para aliviar os sintomas adversos da quimioterapia também estão associadas a efeitos colaterais, toxicidade e dependência, representando um grande desafio para os prestadores de cuidados com o câncer. Portanto, os pacientes tendem a usar terapias não farmacológicas. Diante disso, um estudo demonstra que a prática da yoga ajuda na diminuição da fadiga durante o tratamento quimioterápico, o que colabora positivamente na melhora da qualidade de vida dos pacientes pediátricos. As intervenções de yoga mostraram-se benéficas e produziram resultados positivos sem resultados adversos (Agarwal \& Maroko-Afek, 2018).

Outra intervenção não farmacológica utilizada durante o tratamento do câncer pediátrico é a atividade física. Atualmente, considera-se que muita imobilidade pode resultar em uma diminuição adicional da aptidão física e do funcionamento físico. Esses efeitos adversos podem ser evitados ou minimizados com a introdução de uma intervenção de treinamento físico durante ou logo após o tratamento do câncer infantil (Johnston, et.al., 2015; Mitchell, et.al., 2016). O uso do exercício físico no tratamento quimioterápico pode levar à percepções mais favoráveis de qualidade de vida, ainda que estejam em situação de vulnerabilidade patológica (Johnston, et.al., 2015).

\section{Conclusão}

As evidências mostram que existem fatores que influenciam negativamente na qualidade de vida de crianças submetidas à quimioterapia. No entanto, ainda é necessário mais estudos sobre essa temática, para que se possa desenvolver melhor um tratamento que contribua para a melhora da qualidade de vida dos pacientes. Durante a pesquisa, houve dificuldades em encontrar estudos científicos atuais relacionados ao assunto, visto que as publicações eram antigas, alguns artigos chineses abordavam o tema, enquanto artigos americanos e brasileiros focavam na QV do cuidador da criança com câncer. 
No contexto dos estudos científicos, é relevante o conhecimento sobre os fatores que influenciam na QV das crianças submetidas à quimioterapia, tornando-se essencial para realizar um cuidado com intervenções eficazes de qualidade. Diante da análise desta revisão, observou-se a necessidade da publicação de novos artigos, bem como de produções brasileiras atualizadas acerca do tema, visando proporcionar uma melhora na qualidade de vida das crianças em tratamento quimioterápico e traçar estratégias que ajudem a reduzir os efeitos adversos causado pela quimioterapia.

\section{Referências}

Agarwal, R. P., \& Maroko-Afek, A. (2018). Yoga into cancer care: a review of the evidence-based research. International Journal of Yoga, 11(1), 3-29.

Almico, T., \& Faro, A. (2014). Enfrentamento de cuidadores de crianças com câncer em processo de quimioterapia. Psicologia, Saúde e Doenças, 15(3), 723737.

Andersen, B. L., DeRubeis, R. J., Berman, B. S., Gruman, J., Champion, V. L., Massie, M. J., Holland, J. C., Partridge, A. H., Bak, K., Somerfield, M. R., \& Rowland, J. H. (2014). Screening, assessment, and care of anxiety and depressive symptoms in adults with cancer: an American Society of Clinical Oncology guideline adaptation. Journal of Clinical Oncology, 32(15), 1605-1619.

Barros, L. F., Santos, C. J. D. O., Moro, T. N. P., \& de Jesus, V. M. F. (2017). Estudo de revisão da qualidade de vida e câncer infanto juvenil. Revista Rede de Cuidados em Saúde, 10(1), 1-13.

Braam, K. I., Van der Torre, P., Takken, T., Veening, M. A., Van Dulmen-den Broeder, E., \& Kaspers, G. J. (2016). Physical exercise training interventions for children and young adults during and after treatment for childhood cancer. Cochrane database of systematic reviews, 3(3).

Brasil. (2016). Ministério da Saúde. Instituto Nacional de Câncer José Alencar Gomes da Silva. Incidência, mortalidade e morbidade hospitalar por câncer em crianças, adolescentes e adultos jovens no Brasil: informações dos registros de câncer e do sistema de mortalidade. https://www.inca.gov.br/sites/ufu.sti.inca.local/files//media/document//incidencia-mortalidade-morbidade-hospitalar-por-cancer.pdf

Castro, E. H. B. (2010). A experiência do câncer infantil: repercussões familiares, pessoais e sociais. Revista Mal-estar e Subjetividade, 10(3), 971-994.

Vries, M. A., Van Litsenburg, R. R., Huisman, J., Grootenhuis, M. A., Versluys, A. B., Kaspers, G. J. L., \& Gemke, R. J. (2008). Effect of dexamethasone on quality of life in children with acute lymphoblastic leukaemia: a prospective observational study. Health and Quality of Life Outcomes, 6(1), 103.

Elias, J. S., Moreira, N. D., \& Parra, C. R. (2017). A importância do brincar na hospitalização de crianças com câncer. Recuperado em 15 abril, 2021, de https://www.psicologia.pt/artigos/textos/A1121.pdf

Eiser, C., Stride, C. B., Vora, A., Goulden, N., Mitchell, C., Buck, G., \& National Cancer Research Institute Childhood Leukaemia Sub-Group and UK Childhood Leukaemia Clinicians Network. (2017). Prospective evaluation of quality of life in children treated in UKALL 2003 for acute lymphoblastic leukaemia: A cohort study. Pediatric Blood e Cancer, 64(11), e26615.

Felix-Ukwu, F., Reichert, K., Bernhardt, M. B., Schafer, E. S., \& Berger, A. (2018). Evaluation of aprepitant for acute chemotherapy-induced nausea and vomiting in children and adolescents with acute lymphoblastic leukemia receiving high-dose methotrexate. Pediatric Blood e Cancer, 65 (2), e26857.

Fermo, V. C., Lourençatto, G. N., Medeiros, T. S., Anders, J. C., \& Souza, A. I. J. (2014). O diagnóstico precoce do câncer infantojuvenil: o caminho percorrido pelas famílias. Escola Anna Nery, 18(1), 54-59.

Galvão, T. F., Pansani, T. S. A., \& Harrad, D. (2015). Principais itens para relatar Revisões sistemáticas e Meta-análises: A recomendação PRISMA. Epidemiologia e Serviços de Saúde, 24(2), 335-342.

Mendes, K. D. S., Silveira, R. C. D. C. P., \& Galvão, C. M. (2008). Revisão integrativa: método de pesquisa para a incorporação de evidências na saúde e na enfermagem. Texto e Contexto-Enfermagem, 17(4), 758-764.

Giagnuolo, G., Buffardi, S., Rossi, F., Petruzziello, F., Tortora, C., Buffardi, I., Marra, N., Beneduce, G., Menna G., \& Parasole, R. (2019). Single center experience on efficacy and safety of Aprepitant for preventing chemotherapy-induced nausea and vomiting (CINV) in pediatric Hodgkin Lymphoma. PloS one, 14(4), e0215295.

Hinds, P. S., Wang, J., Stern, E. D., Macpherson, C. F., Wharton, C. M., Okorosobo, R., Cheng, Y. I., Gross H. E., Meany, H. J., \& Jacobs, S. (2017). Voices of children and adolescents on phase 1 or phase 2 cancer trials: A new trial endpoint?. Cancer, 123(19), 3799-3806.

Johnston, D., Gerbing, R., Alonzo, T., Aplenc, R., Nagarajan, R., Schulte, F., Cullen, P., \& Sung, L. (2015). Patient-reported outcome coordinator did not improve quality of life assessment response rates: a report from the Children's Oncology Group. PloS one, 10(4), e0125290.

Klaassen, R. J., Mathias, S. D., Buchanan, G., Bussel, J., Deuson, R., Young, N. L., Collier, A., Bomgaars, L., \& Blanchette, V. (2012). Pilot study of the effect of romiplostim on child health-related quality of life (HRQoL) and parental burden in immune thrombocytopenia (ITP). Pediatric blood \& cancer, 58(3), $395-398$.

Matos, T. D. S., Meneguin, S., Ferreira, M. L. S., \& Miot, H. A. (2017). Qualidade de vida e coping religioso-espiritual em pacientes sob cuidados paliativos oncológicos. Revista Latino-Americana de Enfermagem, 25, e2910. https://doi.org/10.1590/1518-8345.1857.2910

Melnyk, B. M., \& Fineout-Overholt, E. (Eds.). (2011). Evidence-based practice in nursing \& healthcare: A guide to best practice. Lippincott Williams \& Wilkins.

Mitchell, H. R., Lu, X., Myers, R. M., Sung, L., Balsamo, L. M., Carroll, W. L., Raetz, E., Loh, M. L., Mattano, L. A., Jr, Winick, N. J., Devidas, M., Hunger, S. P., Maloney, K., \& Kadan-Lottick, N. S. (2016). Prospective, longitudinal assessment of quality of life in children from diagnosis to 3 months off treatment 
Research, Society and Development, v. 10, n. 11, e547101119946, 2021

(CC BY 4.0) | ISSN 2525-3409 | DOI: http://dx.doi.org/10.33448/rsd-v10i11.19946

for standard risk acute lymphoblastic leukemia: Results of Children's Oncology Group study AALL0331. International journal of cancer, 138(2), 332-339. https://doi.org/10.1002/ijc.29708

Monteiro, A. C. M., Rodrigues, B. M. R. D., de Araújo Pacheco, S. T., \& Pimenta, L. S. (2014). A atuação do enfermeiro junto à criança com câncer: cuidados paliativos [Nurses' work with children with cancer: palliative care]. Revista Enfermagem UERJ, 22(6), 778-783.

Morais, G. S. D. N., Costa, S. F. G. D., França, J. R. F. D. S., Duarte, M. C. S., Lopes, M. E. L., \& Batista, P. S. D. S. (2018). Experiência existencial de crianças em tratamento quimioterápico sobre a importância do brincar. Revista da Rede de Enfermagem do Nordeste, 19, 1-8.

National Comprehensive Cancer Network. (2013). NCCN clinical practice guidelines in oncology: cancer-related fatigue. National Comprehensive Cancer Network, Version,1.

Orme, L. M., Babl, F. E., Barnes, C., Barnett, P., Donath, S., \& Ashley, D. M. (2014). Outpatient versus inpatient IV antibiotic management for pediatric oncology patients with low risk febrile neutropenia: a randomised trial. Pediatric blood \& cancer, 61(8), 1427-1433.

Richter, D., Mehnert, A., Schepper, F., Leuteritz, K., Park, C., \& Ernst, J. (2018). Validation of the German version of the late adolescence and young adulthood survivorship-related quality of life measure (LAYA-SRQL). Health and quality of life outcomes, 16(1), 4. https://doi.org/10.1186/s12955-017$0827-1$

Rodrigues, A. B., Martin, L. G. R.; Moraes, M. W. (2016). Oncologia Multiprofissional - Serie Manuais De Especialização. Manole.

Ryerson, A. B., Wasilewski-Masker, K., Border, W. L., Goodman, M., Meacham, L., Austin, H., Marchak, J. G., \& Mertens, A. C. (2016). Pediatric quality of life in long-term survivors of childhood cancer treated with anthracyclines. Pediatric blood \& cancer, 63(12), 2205-2211.

Sands, S., Ladas, E. J., Kelly, K. M., Weiner, M., Lin, M., Ndao, D. H., Dave, A., Vahdat, L. T., \& Bender, J. G. (2017). Glutamine for the treatment of vincristine-induced neuropathy in children and adolescents with cancer. Supportive care in cancer: official journal of the Multinational Association of Supportive Care in Cancer, 25(3), 701-708.

Santos, S., Crespo, C., Canavarro, M. C., Fernandes, A., Batalha, L., de Campos, D., \& Pinto, A. (2016). Psychometric study of the European Portuguese version of the PedsQL 3.0 Cancer Module. Health and quality of life outcomes, 14(1), 20.

Shi, X., Tian, L., Zhu, X. D., Wang, H. M., \& Qin, H. (2011). Effect of Chinese drugs combining with chemotherapy on quality of life in 146 children with solid tumor. Chinese journal of integrative medicine, 17(1), 31-34.

Souza, J. A. F., Alves, J. T., Chamma, B. M., Moussa, L., \& Mendes, M. R. P. (2017). Atuação da fisioterapia no controle da dor no câncer infantil. Revista Pesquisa e Ação, 3(2), 73-83.

World Health Organization. (1995). World Health Organization quality of life assessment (The WHOQOL): position paper from the World Health Organization. Social Science \& Medicine. https://www.ncbi. nlm.nih.gov/pubmed/8560308

Vieira, Sheila de Souza, Dupas, Giselle, \& Ferreira, Noeli Marchioro Liston Andrade. (2009). Doença renal crônica: conhecendo a experiência da criança. Escola Anna Nery, 13(1), 74-83. https://doi.org/10.1590/S1414-81452009000100011 